International Journal of Engineering \& Technology, $7(2.33)(2018) 466-469$
International Journal of Engineering \& Technology
WPC

\title{
Image contrast enhancement techniques-a survey
}

\author{
V.S. Padmavathy ${ }^{1}$, Dr. R. Priya ${ }^{2}$ \\ ${ }^{1}$ Research Scholar, Department of Computer Applications, VISTAS, Chennai, India \\ ${ }^{2}$ Associate Professor, Department of Computer Applications, VISTAS, Chennai, India \\ *Corresponding author E-mail: vspadmavathy@gmail.com
}

\begin{abstract}
Image Enhancement plays an essential role in a wide area of vision applications. Image enhancement is a technique used to enhance the qual-ity of the image such that it can be easily viewed by both men and machine.Contrast makes a visual difference that makes an object distin-guishable from background and other objects. The major goal of image contrast enhancement is to increase the visual quality of the image. In this research study, various image contrast enhancement techniques are reviewed. This research work also focuses on the comparative study of contrast enhancement techniques for identifying an effective contrast enhancement technique.
\end{abstract}

Keywords: Image Enhancement; Contrast Enhancement; Spatial Domain; Frequency Domain.

\section{Introduction}

Image processing is an advanced technique by which a low quality image can be converted to a high quality image digitally such that important information can be retrieved from the same. Experts alter the image information through Image enhancement techniques which play a vital role in image processing applications.Image enhancement includes dominance of certain properties or suppressing the ambiguity of two different regions of the image.
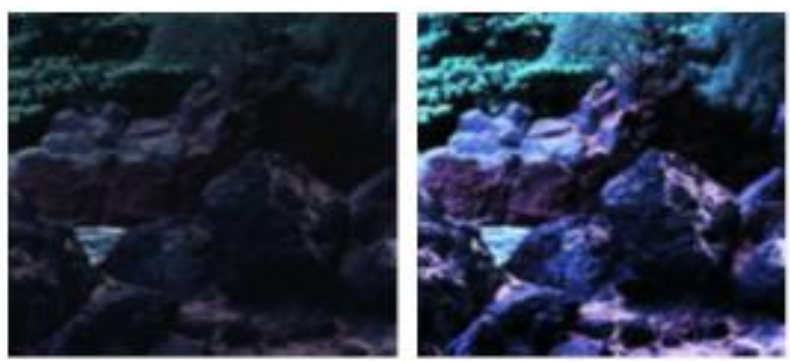

Fig. 1: Enhancement Results A) Original Image B) Enhanced Image D.

\subsection{Image enhancement techniques}

Image enhancement technique includes both Spatial domain methods. and Frequency domain methods. Whereas Spatial domain method directly works upon the pixel value to obtain better enhancement. Point processing methods, Gray Level Transformation, log transformation, histogram processing, Image Negatives ,morphological operators, Piecewise Linear Transformation, Global Power Law Transform, Adaptive Power Law Transform, Spatial Filtering are spatial domain enhancement methods.

Frequency domain method basically works on image transform coefficients like Fourier Transform (FT), Discrete Cosine Transform (DCT), Discrete Wavelet Transform (DWT). In Frequency Domain method, the Fourier transform coefficient is computed first followed by inverse Fourier transform in order to obtain the perfection.

\subsection{Contrast enhancement techniques}

Contrast Enhancement aims at enhancing the global or local contrast of an image. Some of the enhancement techniques include histogram equalization, Genetic algorithms and fuzzy set algorithms. Many modifications are done in the standard methods to improve the contrast of the images.

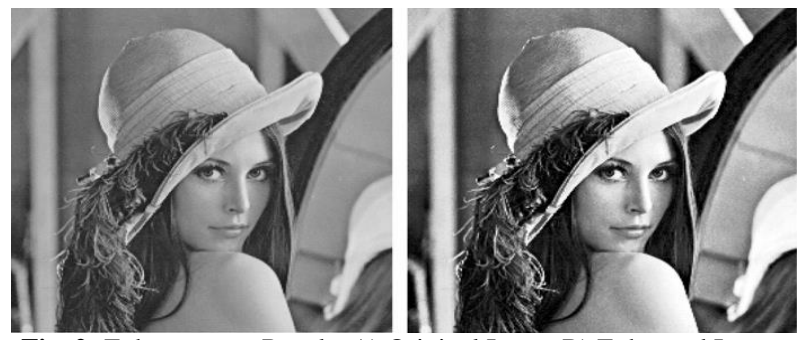

Fig. 2: Enhancement Results A) Original Image B) Enhanced Image.

\section{Literature survey}

M. Shakeri [6] proposed a contrast enhancement algorithm based on local histogram equalization which was used to calculate the total sub-histograms automatically and their division based on their density.The algorithm worked in three stages. Initially, the estimation of the number of clusters for image brightness levels is done using histogram equalization. In the next stage, the image brightness levels are clustered and finally include the contrast enhancement for each individual cluster separately. The algorithm is compared with other methods based on quality and quantity measurement.

Lalit Maurya [7] proposed a social spider optimization algorithm which produces two quality images one with better contrast, increased entropy and the second image with increased peak signal to noise ratio.Both the images are combined to get an effective image later. Comparisons were done with HE, Linear contrast stretching, 
Standard Particle Swarm Optimization. Results show that the proposed method achieves better Peak signal to noise ratio, preserves brightness, enhances the contrast of any given image resulting a quality visual.

Se EunKim [8] worked upon wavelet domain using entropy for contrast enhancement.Initially it uses a local entropy scaling in the wavelet domain to obtain the desired contrast .mathematical works were used and then a color enhancing method in the HSI color space was developed. The algorithm worked in two steps: Modification of the low frequencies in the wavelet domain and scaling HIS colour space by enhancing the intensity component such that low light images will get detailed colour information with out any post processing.

Anil Singh Parihar [9] proposes an entropy-based dynamic sub-histogram equalization algorithm for contrast enhancement with better intensity levels over the entire dynamic range.Parameters were not used.Results were compared with conventional contrast enhancement algorithms.

Jeyong Shin [10] proposed HBLPCE,an optimzization problem to preserve the localities of the histogram for performing contrast enhancement. By this method the shape of the enhanced image remains the same as the original image. HBLPCE was quite successful on all images with different statistical properties.

Huang Lidong [11] proposed an image enhancement method CLAHE-DWT which combines both CLAHE and DWT. The algorithm works in three stages. First the original image is disposed to as low frequency and high frequency components byDWT.Low frequency coefficients are enhanced using CLAHE and high frequency coefficients are unchanged.Upon Inverse DWT the image is mounted successfully.LEI, Noise estimation, PSNR, MAE are the parameters used for evaluation. Results show impressive performance and the over enhancement could be avoided.
Mayank Tiwari [12] proposed a highspeed quantile-based histogram equalisation (HSQHE) algorithm for contrast enhancement suitable for high contrast digital images. The recursive segmentation of the histogram is not done, so only a minimal time is required for segmentation.For the Assessment of contrast enhancement PSNR, Entropy metrics are used. For Assessment of brightness preservation AMBE is used. HSQHE preserves image brightness more accurately in less time interval.

Zhao Wei [13] proposed a EMHM (Entropy maximization histogram modification) method, which consists of dividing the global histogram equalization into two steps, pixel populations mergence (PPM) step which goes in hand with entropy maximisation rule followed by the grey-levels distribution (GLD). Proposed method performs better than the existing methods.

Mohsen Abdoli [14] proposed a new contrast enhancement method named GMMCE (Gaussian mixture model-based contrast enhancement) to enhance low contrast images. This method deals with the histogram of low-contrast image using Gaussian which represents dominant intensity level of the image. Upon enhancing the sub histogram separated by the mean value of the Gaussians of the GMM the global contrast enhancement of the image is achieved.Experimental results show that the shape preserving method of GMMCE enhances the contrast of the image.

Kristofor B [15] proposed CETM to improve the turbulence removal algorithm. An analysis of fog and turbulence is incorporated in this method.Turbulence Mitigation Metric (TMM) is also proposed to evaluate turbulence. The average of the motion compensated images is considered in order to remove the turbulent artifacts. Comparative Study of different Contrast Enhancement techniques.

Table 1: Comparative Study of Different Contrast Enhancement Techniques

\begin{tabular}{|c|c|c|c|c|c|c|}
\hline S. No. & Authors & Title of the paper & Methodology & Features & Advantages & Disadvantages \\
\hline 1 & $\begin{array}{l}\text { Huanjing Yue, } \\
\text { Jingyu Yang, } \\
\text { Xiaoyan Sun, } \\
\text { Feng Wu }\end{array}$ & $\begin{array}{l}\text { Contrast Enhancement } \\
\text { Based on Intrinsic Image } \\
\text { Decomposition, } 2017\end{array}$ & $\begin{array}{l}\text { Split Bregman } \\
\text { algorithm and } \\
\text { CLAHE }\end{array}$ & $\begin{array}{l}\text { To enhance images by } \\
\text { estimating illumination } \\
\text { and reflectance layers } \\
\text { through intrinsic image } \\
\text { decomposition }\end{array}$ & $\begin{array}{l}\text { Good enhance- } \\
\text { ment }\end{array}$ & $\begin{array}{l}\text { Designed only for } \\
\text { CE. Cannot be used } \\
\text { for methods like sur- } \\
\text { face re-texturing, ob- } \\
\text { ject insertion etc }\end{array}$ \\
\hline 2 & $\begin{array}{l}\text { Cheolkon Jung, } \\
\text { Tingting Sun }\end{array}$ & $\begin{array}{l}\text { Optimized Perceptual } \\
\text { Tone Mapping for Con- } \\
\text { trast Enhancement of } \\
\text { Images, } 2017\end{array}$ & $\begin{array}{l}\text { Optimized Per- } \\
\text { ceptual Tone } \\
\text { Mapping } \\
\text { (OPTM) }\end{array}$ & $\begin{array}{l}\text { Focuses on the human } \\
\text { visual attention by con- } \\
\text { structing a saliency his- } \\
\text { togram and performs } \\
\text { Contrast Enhancement }\end{array}$ & $\begin{array}{l}\text { Improves the } \\
\text { performance } \\
\text { without over } \\
\text { enhancement }\end{array}$ & $\begin{array}{l}\text { Needs more time for } \\
\text { CE compared to } \\
\text { HE,CLAHE }\end{array}$ \\
\hline 3 & $\begin{array}{l}\text { Daeyeong Kim, } \\
\text { Changick Kim }\end{array}$ & $\begin{array}{l}\text { Contrast Enhancement } \\
\text { Using Combined 1-D } \\
\text { and 2-D Histogram- } \\
\text { Based Techniques,2017 }\end{array}$ & $\begin{array}{l}\text { Histogram } \\
\text { stretching tech- } \\
\text { nique ,quadratic } \\
\text { programming }\end{array}$ & $\begin{array}{l}\text { To preserve the shape of } \\
\text { the 1-D histogram the } \\
\text { statistical information } \\
\text { on the gray-level differ- } \\
\text { ences }\end{array}$ & $\begin{array}{l}\text { Enhanced im- } \\
\text { ages and per- } \\
\text { ceptual image } \\
\text { quality }\end{array}$ & $\begin{array}{l}\text { Processing time is } \\
\text { slower }\end{array}$ \\
\hline 4 & $\begin{array}{l}\text { Anil Singh Pari- } \\
\text { har, Om Prakash } \\
\text { Verma, Chintan } \\
\text { Khanna }\end{array}$ & $\begin{array}{l}\text { Fuzzy-Contextual Con- } \\
\text { trast Enhancement,2017 }\end{array}$ & $\begin{array}{l}\text { Fuzzy dissimi- } \\
\text { larity histogram } \\
\text { (FDHE), Fuzzy } \\
\text { Contextual Con- } \\
\text { trast Enhance- } \\
\text { ment (FCCE) }\end{array}$ & $\begin{array}{l}\text { Captures the intensity } \\
\text { level differences in the } \\
\text { neighborhood of the } \\
\text { pixels }\end{array}$ & $\begin{array}{l}\text { Global and lo- } \\
\text { cal CE. } \\
\text { No parameters } \\
\text { are used. } \\
\text { Original shape } \\
\text { of histogram is } \\
\text { preserved }\end{array}$ & EME measure is low \\
\hline 5 & $\begin{array}{l}\text { Shilpa Suresh, } \\
\text { Shyam Lal, } \\
\text { Chintala } \\
\text { Sudhakar } \\
\text { Reddy, Mustafa } \\
\text { Servet Kiran }\end{array}$ & $\begin{array}{l}\text { A Novel Adaptive } \\
\text { Cuckoo Search Algo- } \\
\text { rithm for Contrast En- } \\
\text { hancement of Satellite } \\
\text { Images, } 2017\end{array}$ & $\begin{array}{l}\text { Novel Adaptive } \\
\text { cuckoo search } \\
\text { based enhance- } \\
\text { ment algorithm } \\
\text { (ACSEA) }\end{array}$ & $\begin{array}{l}\text { Contrast enhancement } \\
\text { for satellite images }\end{array}$ & $\begin{array}{l}\text { Improved con- } \\
\text { vergence rate. } \\
\text { Good efficiency } \\
\text { and robustness }\end{array}$ & $\begin{array}{l}\text { Complex in its exe- } \\
\text { cution }\end{array}$ \\
\hline 6 & $\begin{array}{l}\text { M.Shakeri, } \\
\text { M.H.Dezfoulian, } \\
\text { H.Khotanlou, } \\
\text { A.H.Barati, } \\
\text { Y.Masoumi }\end{array}$ & $\begin{array}{l}\text { Image contrast enhance- } \\
\text { ment using fuzzy clus- } \\
\text { tering with adaptive } \\
\text { cluster parameter and } \\
\text { sub-histogram equaliza- } \\
\text { tion, } 2017\end{array}$ & $\begin{array}{l}\text { Contrast en- } \\
\text { hancement algo- } \\
\text { rithm based on } \\
\text { local histogram } \\
\text { equalization }\end{array}$ & $\begin{array}{l}\text { Determination of the } \\
\text { number of sub-histo- } \\
\text { grams and density based } \\
\text { histogram division }\end{array}$ & $\begin{array}{l}\text { Natural appear- } \\
\text { ance of images } \\
\text { and enhanced } \\
\text { the contrast }\end{array}$ & $\begin{array}{l}\text { Loss of details in } \\
\text { high brightness levels } \\
\text { of the image. } \\
\text { Noise in the output } \\
\text { image. }\end{array}$ \\
\hline 7 & $\begin{array}{l}\text { Lalit Maurya, } \\
\text { Prasant Kumar } \\
\text { Mahapatra, } \\
\text { Amod Kumar }\end{array}$ & $\begin{array}{l}\text { A social spider opti- } \\
\text { mized image fusion ap- } \\
\text { proach for contrast en- } \\
\text { hancement and bright- } \\
\text { ness preservation, } 2017\end{array}$ & $\begin{array}{l}\text { A social spider } \\
\text { optimization } \\
\text { (SSO)algorithm }\end{array}$ & $\begin{array}{l}\text { Improvement in sharp- } \\
\text { ness, PSNR, brightness } \\
\text { preservation }\end{array}$ & $\begin{array}{l}\text { Better visual } \\
\text { quality }\end{array}$ & $\begin{array}{l}\text { The number of edge } \\
\text { pixels of HE tech- } \\
\text { nique is high while } \\
\text { the fitness value is } \\
\text { less }\end{array}$ \\
\hline
\end{tabular}




\begin{tabular}{|c|c|c|c|c|c|c|}
\hline 8 & $\begin{array}{l}\text { Se } \\
\text { EunKim,JongJu } \\
\text { Jeon,IlKyuEom }\end{array}$ & $\begin{array}{l}\text { Image contrast enhance- } \\
\text { ment using entropy scal- } \\
\text { ing in wavelet do- } \\
\text { main, } 2016\end{array}$ & $\begin{array}{l}\text { An entropy } \\
\text { based contrast } \\
\text { enhancement } \\
\text { method in the } \\
\text { wavelet domain }\end{array}$ & $\begin{array}{l}\text { Used in HSI color space } \\
\text { and performs image } \\
\text { contrast enhancement }\end{array}$ & $\begin{array}{l}\text { Color infor- } \\
\text { mation of low } \\
\text { light images are } \\
\text { good without } \\
\text { any post pro- } \\
\text { cessing. }\end{array}$ & $\begin{array}{l}\text { Over-enhanced re- } \\
\text { gions exist }\end{array}$ \\
\hline 9 & $\begin{array}{l}\text { Anil Singh Pari- } \\
\text { har, Om Pra- } \\
\text { kash Verma }\end{array}$ & $\begin{array}{l}\text { Contrast enhancement } \\
\text { using entropy-based dy- } \\
\text { namic sub-histogram } \\
\text { equalization, } 2016\end{array}$ & $\begin{array}{l}\text { Entropy-based } \\
\text { dynamic sub- } \\
\text { histogram equal- } \\
\text { ization algo- } \\
\text { rithm (EDSHE) }\end{array}$ & $\begin{array}{l}\text { Performs a recursive di- } \\
\text { vision of the histogram } \\
\text { based on the entropy of } \\
\text { the sub histograms }\end{array}$ & $\begin{array}{l}\text { Natural-look- } \\
\text { ing, good con- } \\
\text { trast images }\end{array}$ & $\begin{array}{l}\text { Entropy measure is } \\
\text { less for few images. }\end{array}$ \\
\hline 10 & $\begin{array}{l}\text { Jeyong Shin, } \\
\text { Rae-Hong Park }\end{array}$ & $\begin{array}{l}\text { Histogram-Based Local- } \\
\text { ity-Preserving Contrast } \\
\text { Enhancement, } 2015\end{array}$ & $\begin{array}{l}\text { Histogram- } \\
\text { based locality- } \\
\text { preserving CE } \\
\text { (HBLPCE) }\end{array}$ & $\begin{array}{l}\text { To preserve the locali- } \\
\text { ties of the histogram for } \\
\text { performing contrast en- } \\
\text { hancement }\end{array}$ & $\begin{array}{l}\text { Adapts well on } \\
\text { images with } \\
\text { various statisti- } \\
\text { cal properties. }\end{array}$ & $\begin{array}{l}\text { Longer Execution } \\
\text { time of global CE for } \\
\text { small images. }\end{array}$ \\
\hline 11 & $\begin{array}{l}\text { Huang Lidong, } \\
\text { Zhao Wei, } \\
\text { Wang Jun, Sun } \\
\text { Zebin }\end{array}$ & $\begin{array}{l}\text { Combination of contrast } \\
\text { limited adaptive histo- } \\
\text { gram equalisation and } \\
\text { discrete wavelet trans- } \\
\text { form for image enhance- } \\
\text { ment,2015 }\end{array}$ & $\begin{array}{l}\text { Combines both } \\
\text { CLAHE and } \\
\text { DWT }\end{array}$ & $\begin{array}{l}\text { To enhance the local de- } \\
\text { tails of an image }\end{array}$ & $\begin{array}{l}\text { Performs well } \\
\text { in detail preser- } \\
\text { vation and } \\
\text { noise suppres- } \\
\text { sion. }\end{array}$ & $\begin{array}{l}\text { High-frequency com- } \\
\text { ponent which con- } \\
\text { tains most of } \\
\text { the noise in original } \\
\text { image is unchanged }\end{array}$ \\
\hline 12 & $\begin{array}{l}\text { Mayank Tiwari, } \\
\text { Bhupendra } \\
\text { Gupta, Manish } \\
\text { Shrivastava }\end{array}$ & $\begin{array}{l}\text { High-speed quantile- } \\
\text { based histogram equali- } \\
\text { sation for brightness } \\
\text { preservation and contrast } \\
\text { enhancement, } 2015\end{array}$ & $\begin{array}{l}\text { Highspeed } \\
\text { quantile-based } \\
\text { histogram equal- } \\
\text { isation } \\
\text { (HSQHE) }\end{array}$ & $\begin{array}{l}\text { Contrast enhancement } \\
\text { suitable for high con- } \\
\text { trast digital images }\end{array}$ & $\begin{array}{l}\text { Image bright- } \\
\text { ness more accu- } \\
\text { rately in less } \\
\text { time interval }\end{array}$ & $\begin{array}{l}\text { High PSNR value } \\
\text { only for certain im- } \\
\text { ages }\end{array}$ \\
\hline 13 & $\begin{array}{l}\text { Zhao Wei, } \\
\text { Huang Lidong, } \\
\text { Wang Jun, Sun } \\
\text { Zebin }\end{array}$ & $\begin{array}{l}\text { Entropy maximisation } \\
\text { histogram modification } \\
\text { scheme for image en- } \\
\text { hancement, } 2015\end{array}$ & $\begin{array}{l}\text { Entropy maxi- } \\
\text { mization histo- } \\
\text { gram modifica- } \\
\text { tion } \\
(\mathrm{EMHM})\end{array}$ & $\begin{array}{l}\text { Divides the global histo- } \\
\text { gram equalization into } \\
\text { two steps, pixel popula- } \\
\text { tions mergence (PPM) } \\
\text { step and the grey-levels } \\
\text { distribution (GLD) step }\end{array}$ & $\begin{array}{l}\text { Good } \\
\text { enhancement, } \\
\text { avoids ampli- } \\
\text { fied noise and } \\
\text { image artefacts. }\end{array}$ & $\begin{array}{l}\text { Contrast Overstretch- } \\
\text { ing problem }\end{array}$ \\
\hline 14 & $\begin{array}{l}\text { Mohsen Abdoli, } \\
\text { Hossein } \\
\text { Sarikhani, Mo- } \\
\text { hammad Ghan- } \\
\text { bari, Patrice } \\
\text { Brault }\end{array}$ & $\begin{array}{l}\text { Gaussian mixture model- } \\
\text { based contrast Enhance- } \\
\text { ment, } 2015\end{array}$ & $\begin{array}{l}\text { Gaussian mix- } \\
\text { ture model- } \\
\text { based contrast } \\
\text { enhance- } \\
\text { ment)(GMMCE) }\end{array}$ & $\begin{array}{l}\text { Uses Gaussian mixture } \\
\text { modeling of histograms } \\
\text { to model the content of } \\
\text { the images }\end{array}$ & $\begin{array}{l}\text { Lowest approx- } \\
\text { imation error } \\
\text { highest similar- } \\
\text { ity to the origi- } \\
\text { nal histogram } \\
\text { low-complexity } \\
\text { method }\end{array}$ & $\begin{array}{l}\text { Subjective Quality } \\
\text { measures not used }\end{array}$ \\
\hline 15 & $\begin{array}{l}\text { Kristofor B. } \\
\text { Gibson and Tru- } \\
\text { ong Q. Nguyen }\end{array}$ & $\begin{array}{l}\text { An Analysis and Method } \\
\text { for Contrast Enhance- } \\
\text { ment Turbulence Mitiga- } \\
\text { tion, } 2014\end{array}$ & $\begin{array}{l}\text { Contrast en- } \\
\text { hancement and } \\
\text { turbulence miti- } \\
\text { gation (CETM) }\end{array}$ & $\begin{array}{l}\text { Provides an analysis of } \\
\text { fog turbulence }\end{array}$ & $\begin{array}{l}\text { Increased con- } \\
\text { trast } \\
\text { Less time con- } \\
\text { sumption }\end{array}$ & PSNR is very low \\
\hline
\end{tabular}

\section{Conclusion}

In this research work, several contrast enhancement techniques were analysed and reviewed. The Comparative study of these techniques is also presented. The investigation of these methods show that some of the methods suffered low processing time, low PSNR, and Entropy values. Few algorithms experienced enhancement only in some regions while the other few had over enhancement, loss of the details in a darker regions etc. In order to overcome these limitations a new approach can be formulated.

\section{References}

[1] Huanjing Yue, Jingyu Yang, Xiaoyan Sun, Feng Wu," Contrast Enhancement Based on Intrinsic Image Decomposition", IEEE TRANSACTIONS ON IMAGE PROCESSING, VOL. 26, NO. 8, pp 3981-3994, AUGUST 2017.

[2] Cheolkon Jung, Tingting Sun,” Optimized Perceptual Tone Mapping for Contrast Enhancement of Images", IEEE TRANSACTIONS ON CIRCUITS AND SYSTEMS FOR VIDEO TECHNOLOGY, VOL 27, NO. 6, 1161-1170, JUNE 2017

[3] Daeyeong Kim, Changick Kim," Contrast Enhancement Using Combined 1-D and 2-D Histogram-Based Techniques", IEEE SIGNAL PROCESSING LETTERS, VOL. 24, NO. 6, 804-808, JUNE 2017.

[4] Anil Singh Parihar, Om Prakash Verma, Chintan Khanna, "FuzzyContextual Contrast Enhancement", IEEE TRANSACTIONS ON IMAGE PROCESSING, VOL. 26, NO. 4, pp. 1810-1819, APRIL 2017.

[5] Shilpa Suresh, Shyam Lal, Chintala Sudhakar Reddy, Mustafa Servet Kiran,"A Novel Adaptive Cuckoo Search Algorithm for Contrast
Enhancement of Satellite Images", IEEE JOURNAL OF SELECTED TOPICS IN APPLIED EARTH OBSERVATIONS AND REMOTE SENSING, IEEE 2017.

[6] M.Shakeri, M.H.Dezfoulian, H.Khotanlou, A.H.Barati, Y.Masoumi, "Image contrast enhancement using fuzzy clustering with adaptive cluster parameter and sub-histogram equalization”, Elsevier Digital signal Processing, pp. 224-237, 2017.

[7] Lalit Maurya, Prasant Kumar Mahapatra, Amod Kumar, "A social spider optimized image fusion approach for contrastenhancement and brightness preservation",Elsevier Applied soft computing, pp. 575-592,2017.

[8] Se EunKim,JongJuJeon,IIKyuEom, "Image contrast enhancement using entropy scaling in wavelet domain”, Elsevier signal Processing, pp. 1-11, 2016.

[9] Anil Singh Parihar, Om Prakash Verma," Contrast enhancement using entropy-based dynamic sub-histogram equalization", IET Image Process., Vol. 10 Iss. 11, pp. 799-808, 2016.

[10] Jeyong Shin, Rae-Hong Park," Histogram-Based Locality-Preserving Contrast Enhancement", IEEE SIGNAL PROCESSING LETTERS, VOL. 22, NO. 9, 1293-1296, SEPTEMBER 2015.

[11] Huang Lidong, Zhao Wei $\square$, Wang Jun, Sun Zebin," Combination of contrast limited adaptive histogram equalisation and discrete wavelet transform for image enhancement", IET Image Processing Journals, Vol. 9, Iss. 10, pp. 908-915, 2015.

[12] Mayank Tiwari, Bhupendra Gupta, Manish Shrivastava," Highspeed quantile-based histogram equalisation for brightness preservation and contrast enhancement", IET Image Process., 2015, Vol. 9, Iss. 1, pp. 80-89.

[13] Zhao Wei, Huang Lidong, Wang Jun, Sun Zebin,” Entropy maximisation histogram modification scheme for image enhancement", IET Image Process., 2015, Vol. 9, Iss. 3, pp. 226-235.

[14] Mohsen Abdoli, Hossein Sarikhani, Mohammad Ghanbari, Patrice Brault," Gaussian mixture model-based contrast Enhancement", IET Image Process., 2015, Vol. 9, Iss. 7, pp. 569-577. 
[15] Kristofor B. Gibson and Truong Q. Nguyen," An Analysis and Method for Contrast Enhancement Turbulence Mitigation", IEEE TRANSACTIONS ON IMAGE PROCESSING, VOL. 23, NO. 7, 3179-3190, JULY 2014.

[16] C. Amiot, C. Girard, J. Chanussot, J. Pescatore, and M. Desvignes," Curvelet Based Contrast Enhancement in Fluoroscopic Sequences", IEEE TRANSACTIONS ON MEDICAL IMAGING, VOL. 34, NO 1, 137-147, JANUARY 2015.

[17] Shilong Liu, Md Arifur Rahman, Ching-Feng Lin, Chin Yeow Wong, Guannan Jiang, San Chi Liu, Ngaiming Kwok, Haiyan Shi,"Image Contrast Enhancement Based on Intensity Expansion-Compression”, Elsevier Journal of Visual Communication \& Image Representation , 2017.

[18] J. Mohan, V. Krishnaveni, Yanhui Guo,"A survey on the magnetic resonance image denoising methods", Elsevier Biomedical Signal Processing and Control, pp. 56-69,2014.

[19] Dr.Muna F. Al-Sammaraie, "Contrast Enhancement of Roads Images with Foggy Scenes Based on Histogram Equalization”, International Conference on Computer Science \& Education (ICCSE 2015) pp.95-101, 2015.

[20] Arash Samani, Karen Panetta, Sos Agaian "Contrast Enhancement for Color Images Using Discrete Cosine Transform Coefficient Scaling",IEEE 2016.

[21] Jinwen Yang,Weihe Zhong,Zheng Miao," On the Image Enhancement histogram Processing", International Conference on Informative and Cybernetics for Computational Social Systems (ICCSS),pp. 252-255, 2016.

[22] Milos Jordanski, Aleksandra Arsic, and Milan Tuba," Dynamic Recursive Subimage Histogram Equalization Algorithm for Image Contrast Enhancement “, IEEE 2015, pp.24-26, 2015. 